\title{
Corela
}

Cognition, représentation, langage

HS-10 | 2012

Paramétrer le sens ? Études de cas

\section{A la recherche des paramètres de l'élaboration du sens au sein des énoncés}

Sarah de Vogüé

\section{(2) OpenEdition}

Journals

Édition électronique

URL : http://journals.openedition.org/corela/2369

DOI : 10.4000/corela.2369

ISSN : 1638-573X

Éditeur

Cercle linguistique du Centre et de l'Ouest - CerLICO

Référence électronique

Sarah de Vogüé, "A la recherche des paramètres de l'élaboration du sens au sein des énoncés ", Corela [En ligne], HS-10 | 2012, mis en ligne le 30 janvier 2012, consulté le 30 avril 2019. URL : http:// journals.openedition.org/corela/2369; DOI : 10.4000/corela.2369

Ce document a été généré automatiquement le 30 avril 2019

\section{(c) (i) (2)(2)}

Corela - cognition, représentation, langage est mis à disposition selon les termes de la licence Creative Commons Attribution - Pas d'Utilisation Commerciale - Partage dans les Mêmes Conditions 4.0 International. 


\title{
A la recherche des paramètres de l'élaboration du sens au sein des énoncés
}

\author{
Sarah de Vogüé
}

\section{Introduction}

1 L'entreprise de paramétrage du sens peut s'inscrire dans deux programmes de recherche différents, selon le type de variation que l'on cherche à prendre en compte au travers de ce paramétrage. Dans celui qui m'occupe, il s'agit de comprendre les mécanismes d'élaboration du sens au sein d'un énoncé, quelle que soit la langue considérée ; l'objectif est donc strictement généralisant, et s'intéresse à des mécanismes qui vont se retrouver mutatis mutandis dans toute langue. Une toute autre perspective serait de rendre compte de la variation de langue à langue, et de chercher à rapporter à des paramètres les différences entre langues pour ce qui concerne la construction du sens : l'objectif est alors plutôt celui d'une linguistique contrastive, qui examine les différences entre langues, et qui cherche non pas seulement à les classer mais à rapporter ces différences à un certain nombre de points de rupture qui seraient les paramètres en question ${ }^{1}$.

2 Je ne m'inscris pas dans cette seconde perspective ici, d'abord parce que je ne crois pas à un modèle de la diversité des langues selon lequel les langues se différencient en classes ou types sémantiques, avec certaines langues relevant dès lors plus de tel ou tel type sémantique (des langues plus subjectives, plus processuelles, etc.) : il y a d'autres modèles de la diversité des langues, dont celui de l'invariance proposé par Antoine Culioli², qui soutient que dans toute langue se retrouvent les mêmes configurations invariantes, qui sont des configurations sans doute variables puisque soumises à variation, mais qui justement rendent compte de cette variation au sens où elles l'intègrent par leur variation même. Ainsi tiendrai-je plutôt que ne varient d'une langue à l'autre que les tours et les détours que prennent ces configurations sémantiques invariantes. 
3 Je m'inscris dans la première perspective ensuite parce que je considère en revanche que les sens qui au travers des énoncés se trouvent élaborés, ne sont pas simplement indéfiniment variés, mais peuvent de fait être caractérisés à la fois dans ce qui les rassemble (on devrait dans cet esprit pouvoir rapporter l'ensemble des sens possibles à une classe : déterminer la classe de ce que peut être un sens pour un énoncé quelle que soit la langue considérée), et dans ce qui les différencie: face aux sens, il est possible d'aller au-delà d'un simple répertoriage, et de reconstituer la façon dont la diversité des sens en question se déploie, la façon dont elle s'organise et dont elle se diversifie.

De ce point de vue, le propos de cet article est de montrer que le paramétrage est un mode de caractérisation pertinent pour rendre compte d'un tel déploiement. Dans une première partie, je me propose de réfléchir sur ce que peut recouvrir ce concept de paramétrage : on examinera en particulier la valeur qui lui est donnée en mathématiques, et on en déduira un certain nombre de caractéristiques qui montrent que le paramétrage ressortit d'une entreprise de formalisation pouvant être au total fort différente de celles qui se présentent d'ordinaire au titre de la sémantique formelle. Dans un second temps, on analysera la façon dont se présente la question générale de la caractérisation du sens tel qu'il s'élabore au travers de l'activité langagière, et on envisagera un certain nombre de paramètres propres à en rendre compte.Les hypothèses que je serai amenée à évoquer ce faisant s'inscrivent pour l'essentiel dans le cadre de la Théorie des Opérations Prédicatives et Enonciatives qui s'est élaborée depuis une trentaine d'années autour des travaux d'Antoine Culioli: des hypothèses dont on peut envisager qu'elles relèvent effectivement d'une entreprise de paramétrage.

\section{Pour une épistémologie du paramétrage}

5 Le terme paramètre, construit à partir du grec parametreô "mesurer (metreô) d'après, par rapport (para : à coté)", vient des mathématiques où il est employé en particulier dans la description des courbes dont on veut déterminer la singularité par une mesure chiffrée : par exemple, si l'on se souvient qu'une parabole est une sorte de cloche dont les bords continueraient à s'écarter indéfiniment, on peut caractériser telle parabole particulière, plus ou moins évasée, par un paramètre, propre à mesurer son degré d'évasement. De manière plus générale, le paramètre est une quantité visant à caractériser une entité mathématique.

6 A partir de là, on le rencontre dans deux domaines, tous deux intéressants pour notre propos: le domaine des statistiques, où il désigne les différentes grandeurs servant à caractériser les ensembles statistiques (médiane, quartile, variance, etc.), et le domaine informatique, où il désigne les données que l'on doit fixer dans le cadre d'une programmation.

7 Par extension, il a un usage courant, selon lequel il désigne un « élément important dont la connaissance explicite les caractéristiques essentielles de l'ensemble d'une question » ( Robert), et partant, plus largement, tout «élément nécessaire pour juger, évaluer, comprendre quelque chose » (Robert). 


\subsection{Efficace}

8 De l'ensemble de ces utilisations du concept, il ressort d'abord que le paramètre se conçoit comme une donnée dotée d'une forme d'efficacité: il est question en programmation informatique d'assurer un «fonctionnement optimal» du programme ( Robert), il est question en statistiques d'exprimer «en abrégé » les caractéristiques d'une série statistique (Turgot, vers 1780, cité par Carbonnel1995), il est question de rapporter un tout à ses caractéristiques essentielles, il est question d'en donner la mesure. Déterminer les éléments propres à expliciter ce qui caractérise ce tout dans son ensemble : il ne s'agit pas seulement d'un mouvement de réduction, il s'agit d'un travail d'explicitation. Et c'est cette explicitation qui se doit d'être efficace: touchant les caractéristiques essentielles du tout en question ${ }^{3}$.

\subsection{Paramétrer le singulier : contre la généralité}

9 Il s'avère par ailleurs que, tout en relevant en plein du domaine mathématique, du moins pour l'essentiel de ses emplois, les autres n'étant que des extensions, le paramètre ne ressortit pas à ce fonctionnement "aveugle » dont Milner(1989) a fait d'une certaine façon le fondement du mathématique. Ou du moins il n'y ressortit pas de la façon habituellement entendue: le paramètre n'est pas de ces «entités dont la référence objective (la substance) [...] n'a pas à être [déterminée] » (Milner1989, p. 91). Le paramètre n'est pas une variable : à la différence des variables, il se doit d'être fixé, donc doté d'une valeur.

10 La différence entre paramètre et variable n'est sans doute pas simple: on en a les manifestations par exemple dans les définitions que tente le Trésor de la Langue Française, quand il allie allègrement les contraires, pour dire que le paramètre est variable mais peut être constant (" variable susceptible de recevoir une valeur constante pour un cas déterminé »), ou quand il évoque « l'ambivalence de ce concept suggérant soit la notion de variable soit celle de constante». On en voit les effets aussi dans les notations formelles utilisées de la manière plus classique (voir les équations ou les fonctions à paramètres qui peuvent être proposées dès les premières classes du lycée), où les paramètres s'écrivent $m$ ou $n$ quand les variables s'écrivent $x, y$ ou $z$, mais paraissent aussi peu fixées les unes que les autres (sauf qu'il s'agit de trouver les solutions de l'équation ou les allures des courbes en fonction des différentes valeurs des paramètres, et non pas en fonction des variables); Carbonnel(1995)cherchant à les utiliser dans le domaine hydraulique qui est le sien, insiste sur la nécessité et la difficulté de les distinguer et propose de tenir pour paramètres les données liées au milieu, plus stables donc, et pour variables les données liées aux alea du temps.

11 Le paramètre doit être fixé, et lorsqu'il l'est, il suffit à caractériser par sa valeur l'essentiel d'un ensemble. Il n'a de variable que le fait qu'il puisse être fixé diversement (qu'il puisse prendre différentes valeurs), justement pour pouvoir caractériser des ensembles variés. S'il est "aveugle ", ce n'est pas au sens où il ne serait pas nécessaire de "voir ce qu'[il] désigne " (Milner,p.91). Le paramètre n'est "aveugle » que dans la mesure où il fait abstraction de tout le reste de l'ensemble dont il n'est que la forme « abrégée », dans la mesure donc où il procède d'une réduction. Encore est-il difficile de parler d'aveuglement là, dans la mesure où justement le paramètre prétend permettre de rendre explicite et 
donc visible ce reste, du moins dans ses caractéristiques essentielles. Si tant est que cette réduction à l'essentiel soit aveuglement, ce qui est difficile à entendre, en tous les cas c'est sur ce reste que porterait l'aveuglement et non pas sur la valeur même du paramètre.

En ce sens, la recherche de paramètres ne s'inscrit pas dans le projet formalisant tel que le définit Russel : il ne s'agit pas de faire abstraction de ce qui serait la « substance » des entités à caractériser pour n'en retenir que la «forme" (voir Russel 1919, p. 234 : «lorsque [...] l'argumentation est formelle, rien ne dépend des termes que l'on y rencontre »). Contrairement à ce qui se passe souvent dans les entreprises formalisantes, il ne s'agit pas de se désintéresser d'une partie de l'empirique (la substance) jugée non pertinente (insuffisamment formelle) ; il s'agit de rendre compte au contraire du tout que forment ces entités, si ce n'est dans leur substance du moins dans leur essence, à coups de paramètres.

13 Par la même occasion, il ne s'agit pas non plus de généraliser, de trouver la formule qui vaudrait pour toute une classe d'empiries singulières (voir le " désir » qu'évoque Russel de ne pas « perdre notre temps à démontrer au particulier ce qui peut être démontré au général »,p.235; voir aussi la conclusion de son argumentation sur la question : « Ainsi l'absence de toute mention de choses ou de propriétés particulières, en logique ou en mathématiques pures, est un résultat nécessaire du fait que cette étude est, comme nous dirons, " purement formelle » »).

Il s'agit tout au contraire de pouvoir rendre compte d'objets empiriques dans leur singularité (ou du moins dans leur essence singulière, si tant est que cette essence soit singulière). Le fait est que ces objets sont des "touts", des "ensembles", ou des programmes informatiques propres à être exécutés en différentes occasions, ou des courbes, intégrant en eux-mêmes des variations. Le propos n'est cependant pas de neutraliser cette variation, mais bien de la rassembler, de la décrire, d'en donner la mesure : un projet orthogonal au projet généralisant.

\subsection{Lettres moins aveugles que maigres}

15 On constate par conséquent avec le paramètre qu'il peut y avoir d'autres «lettres » au sens de Milner que celles qui sont des variables, valent pour leur généralité, et abstraction faite de toute substance propre. S'il s'agit bien toujours de littéralisation, c'est donc en revoyant ce qui est en jeu dans une littéralisation, moins aveugle que résumante.

Cette forme de littéralisation, peut-être est-ce plus chez Frege que chez Russel qu'il faut aller en chercher le modèle, dans l'entreprise même de ce que Frege a appelé Begriffschrift, traduite par "Idéographie ", qui est bien affaire de lettres plutôt que de simples formes. Frege revendique moins la nécessité de faire abstraction des substances individuelles et de généraliser, que celle de la précision, dont il soutient qu'il ne faut jamais qu'elle soit " sacrifiée à la concision de l'expression » (Frege, «Qu'est-ce qu'une fonction? », p. 169). Et de fait, lorsqu'il élabore la Begriffschrift, il s'applique au moins autant à caractériser des fonctionnements, des relations et des «places » qu'à « exprimer la généralité » (p.167,à propos de l'écriture des fonctions et de la différence à faire entre le marquage, par une parenthèse, d'une place, et le marquage par " $\mathrm{x}$ » d'une généralité). L'ambition de cette Idéographie est d'« exprimer immédiatement les pensées au moyen de signes écrits ou imprimés » (ibidem, p. 169), et non pas de faire abstraction de ce qui serait la substance ou la singularité de ces pensées. Sans doute la lettre, pour expliciter au plus près, de la 
manière la plus précise, les pensées qu'elle exprime, doit-elle être la moins médiatisante, la moins substantielle, la moins épaisse possible. Mais c'est de sa substance propre qu'elle doit faire l'économie, et non pas de celle des pensées explicitées : telle que la décrit Frege, la lettre est dépourvue de substance sonore (parce que ce serait un intermédiaire de trop pour exprimer immédiatement les pensées: "sans la médiation du son»); elle est dépourvue de l'ambiguïté propre qui est toujours celle des mots (les mots comme des mains, "diversement habile[s]», Frege 1882, p. 66); elle est sans ces implicites qui peuvent se trouver attachés aux mots et aux phrases (Frege 1882, p. 65); mais c'est d'abord pour être précise («allier à une extrême précision la plus grande concision compatible »,Frege 1904, p. 169), et non pas pour valoir généralement.

\subsection{L'ordre de la lettre : calcul ou invariance}

17 Dans cette précision, on retrouve sans doute l'autre dimension mise en avant par Milner pour faire entendre ce qui serait en jeu dans une littéralisation : qu'elle soit« réservoir » de « contraintes » (Milner, p. 92), aveugles moins au sens là d'une abstraction qu'au sens d'une indifférence aux emplois et donc d'une rigidité et d'une indéformabilité contre toute ambiguïté (Frege 1882, p. 66). Ce qui fait la lettre, c'est sa maigreur d'abord (concise et résumante), mais c'est aussi qu'elle s'inscrive dans une idéographie, avec généralement d'autres lettres, et en tous les cas des règles, des contraintes, un ordre, des emplois, des places, une grammaire, un chiffre. Le paramètre, comme les lettres en général, doit s'inscrire dans un tel ordre, qui fonde sa relation au mathématique.

18 Sur ce plan aussi cependant il faut être prudent quant au sens à donner à cette relation aux contraintes et à la façon dont les lettres en général, les paramètres en particulier, se trouvent ainsi rapportées au champ du mathématique. La lettre emporte avec elle une notion de contrainte, elle n'emporte pas avec elle nécessairement la notion de calcul. Cela est extrêmement important pour notre propos, car cela signifie que si les langues, le langage, le sens sont littéralisables, et en l'occurrence paramétrables, ils ne sont pas nécessairement pour autant calculables: paramétrer le sens ne veut pas dire que l'on pourrait envisager de vouloir le calculer. On mesure l'importance de cette précision quand on sait par ailleurs toutes les entreprises qui s'emploient à un tel calcul et prennent pour principe constituant l'hypothèse de sa possibilitét.

19 Si la lettre est l'instance de contraintes aveugles, d'une grammaire, d'un ordre, elle n'est pas nécessairement le lieu d'un calcul, qui à partir de données détermine de manière contrainte, suivant un ordre déterminé, un résultat.

20 Ce n'est pas nécessaire en tous les cas pour les paramètres: il suffit de parcourir les emplois du terme pour vérifier que le tout que le paramètre explicite, n'est pas toujours calculable. En particulier dans l'emploi statistique du terme, où l'on ne voit pas que les paramètres que sont médianes et écarts-types aient pour fonction de donner les clefs qui rendraient calculable la série qu'ils paramètrent. Il s'agit de caractériser cette série, il n'est pas question de pouvoir la déduire dans ses alea à partir des paramètres.

21 De manière générale, rapportées à des paramètres, les entités empiriques se voient analysées, caractérisées, explicitées dans leurs caractéristiques essentielles, mais ne doivent pas pour autant pouvoir être calculées à partir de ces paramètres, ne doivent pas pouvoir en être déduites dans leur réalité empirique propre. Le paramètre ne ressortit pas à un calcul, encore moins à un calcul qui serait prédictif. 
Si l'empirique paramétré s'inscrit dans un ordre, c'est donc seulement celui de ces caractéristiques essentielles, qui l'ordonnent dans sa singularité. Cet ordre insistant au travers même de la variation, correspond en définitive à ce que Culioli a proposé d'appeler un "invariant ", l'invariant de la variation en question : un invariant qui n'est pas seulement ce qui ne varie pas, qui est ce qui caractérise et ordonne cette variation, qui la caractérise "littéralement " justement, c'est-à-dire "à la lettre ", ou, pour continuer dans le réservoir des expressions qui tentent de faire entendre l'enjeu d'une littéralisation, non pas seulement dans son "esprit ", mais dans le détail même de sa lettre ${ }^{5}$. 'est cet invariant littéral que le paramètre doit restituer.

\subsection{Lettres muettes}

Les « contraintes aveugles » de la littéralisation ne se mesurent pas nécessairement à un calcul, elles se mesurent à l'invariant ordonnant les variations. Si cet invariant est littéral quoiqu'il ne ressortisse pas à un calcul, c'est qu'il répond au critère de précision qu'évoque Frege en sus de la concision pour définir le projet idéographique. Frege parle de clarté logique, de rigueur : l'invariant doit avoir ces propriétés. Pour comprendre ce que cela suppose, on peut prendre au mot ce que Frege en dit dans le passage que nous avons cité : pas de son, pas d'ambiguïté, pas d'implicite. L'ordre idéographique suppose non seulement des lettres maigres (concises), mais des lettres muettes: l'ordre idéographique est sans parole.

Il est en fait même sans parole au sens de Saussure, sans ce qui serait un sujet réalisant, agissant, parlant, maître de sa parole, de ses «habiletés » (voir la référence à la main habile chez Frege), de ses implicites, de la sonorité et des ambiguïtés des signes qu'il manie. Il est ce qui s'écrit sans qu'un sujet agent l'ait écrit ${ }^{6}$. Sans doute en effet est-ce là ce qui, avant le calcul, fonde le mathématique : non pas la généralisation des lois aveugles au réel, mais bien plutôt la rigidité d'un ordre sans sujet agent et maître de ce qui s'écrit . Si le paramètre est une lettre, il faut qu'il s'inscrive ainsi dans l'équivalent de ce qui Saussure s'appelle la langue, à savoir cet ordre dont le sujet agissant a été extrait.

On voit que le projet paramétrant suppose que dans quelque mesure la thèse saussurienne soit entendue, et qu'il soit possible pour ce qui touche au sens et au langage de faire abstraction sinon de toute subjectivité, du moins du sujet agissant qui s'approprie la langue dans une parole qu'il réalise et dont il est le maître.

\subsection{Quantité contre qualité : paramètres et traits}

Le paramètre serait donc bien une lettre, au sens de Frege sinon au sens de Milner: maigre et muette, concise et précise, restituant l'invariant qui s'écrit dans une variation. Mais c'est aussi une donnée, plus précisément une « mesure », ce que toutes les lettres ne sont pas. Car, parler de mesure, c'est se situer dans un domaine quantitatif, et c'est aussi supposer un étalonnage à partir duquel la mesure se trouve évaluée.

Le paramètre est une quantité. Cela ne veut pas dire là qu'il soit nécessairement un nombre, ou même qu'il soit numérisable, contrairement à ce qu'une approche trop rapide de la notion de quantité pourrait laisser à penser. La notion de quantité s'interprète ici par opposition à celle de qualité. Dire que le paramètre est une quantité, c'est dire seulement qu'il procède d'une prédication d'existence plutôt que d'une prédication 
attributive, d'un « il y a x » plutôt que d'un «c'est y ». Cela revient donc à dire que c'est une donnée. quantité qu'est le paramètre, se trouve évaluée et donc fixée en relation à un étalonnage. Il faut par conséquent un tel étalonnage, soit une forme de pré-établi, externe à ce qui va se trouver paramétré. C'est une dernière particularité du paramètre que fait entendre cette fois surtout l'emploi informatique: les paramètres en informatique sont des données de départ, à fixer dans un choix prédéfini, préalable au programme qui doit s'exécuter.

En cela le paramétrage se distingue à nouveau d'autres entreprises littéralisantes : il se distingue de ce qui serait une modélisation, et il se distingue de ce qui serait une formalisation. Quand la modélisation construit un modèle qui reproduit, quand la formalisation reconstitue la forme qui ordonne la substance, le paramétrage n'a pas cette vocation à circonscrire l'empirie. En particulier, au lieu de se situer en surplomb de cette empirie comme le fait le modèle, ou dans une fidélité à sa forme comme cherche à le faire un formalisme, le paramétrage quant à lui, abrégé, antérieur, prédéfini, reste en amont. En ce sens il se trouve associé à un enchaînement. Un enchaînement qui est le contraire de déductif ${ }^{8}$, puisqu'il n'y a pas calcul, qui est un chemin inductif : à partir du choix de 
telle donnée pour paramétrer ce qui a été défini en amont, la variation de l'empirique se déploie.

\subsection{Contre l'épistémologie du réfutable}

On retrouve par conséquent ce que l'on a décrit au départ comme étant l'efficacité du paramètre : un choix de donnée qui suffit à expliciter précisément ce qui fait l'invariant d'une variation.

Cette efficacité, Milner en parle aussi, non pas comme une caractéristique de la littéralisation en soi, mais comme une ambition nécessaire à toute démarche empirique : celle de la « fécondité empirique » (Milner, p. 92).

Dans son système, le garant de cette fécondité est la réfutabilité : la littéralisation doit être réfutable. C'est que la littéralisation qui l'occupe est de fait affaire de valeurs de vérité parce que constituée de propositions, faites en tant que telles pour être vraies ou fausses.

8 Le paramétrage n'est pas énoncé de propositions, mais détermination de mesures à fixer : celles-ci ne sont pas susceptibles d'être vraies ou fausses, donc d'être réfutables; elles sont seulement susceptibles d'être exactes ou pas. Il s'agit qu'elles soient à même de donner la mesure du tout qui se trouve paramétré. Il s'agit qu'elles soient à même de rendre explicites les caractéristiques essentielles de ce tout.

On est ainsi dans une épistémologie qui n'est plus celle directement héritée de Popper, mais qui est plutôt celle qu'a dessinée Lakatos ${ }^{9}$ en donnant les théories comme des programmes d'observation de l'empirique, et les axiomes de départ de ces théories comme des prédications d'existence déterminant les conditions d'observation: dans une telle épistémologie, l'adéquation empirique d'une théorie se mesure directement à sa fécondité -à ce que cette théorie permet de voir. Les paramètres s'évaluent à ce qu'ils rendent visibles.

Ce changement de paradigme épistémologique est important : on sait le rôle joué pour les sciences du langage par le paradigme différentiel qui fonde l'adéquation empirique sur le caractère différentiel des propriétés mises en évidence, que ce soit sous la forme de la théorie de la valeur différentielle dans la doctrine structuraliste, ou au travers de la recherche des inacceptables telle qu'inaugurée par Chomsky pour circonscrire de l'extérieur l'acceptable.

41 Avec les paramètres, on ne cherche pas à différencier tel empirique de tel autre; on cherche d'abord seulement à voir ce dont cet empirique est fait. Il n'est pas nécessaire de procéder par contrastes, oppositions, et différenciations. Il devient alors envisageable que les données soient peu discernées les unes par rapport aux autres, ne se définissent pas par différence. Et il devient envisageable qu'elles ne soient pas stables du coté de l'acceptabilité, que tout dépende du prix qu'on veuille y mettre.

Arriver à voir, à rendre perceptible, et à expliciter ce que sont ces données et ce dont elles sont faites est un objectif suffisant ${ }^{10}$, est même le seul objectif qui vaille puisque aussi bien c'est arriver à comprendre de quoi l'empirique est fait, ce qui est de fait l'objectif premier de la connaissance. 


\subsection{Le paramétrage comme programme épistémologique} langue, ce qui serait donc le sens dans telle langue, mais bien ce qu'est le sens en général, tel du moins qu'il se trouve déployé au travers de cette activité particulière qu'est la production d'énoncés- tous les sens qui peuvent s'y trouver déployés, tous les sens possibles, d'une langue à l'autre. qu'on ne pourrait le penser :on évoquera ici quatre moteurs de diversification du sens, qui sont en fait pour l'essentiel bien connus, mais qui s'avèrent chacun induire un foisonnement de sens tel qu'il ne soit pas possible de fixer des limites au champ recouvert, tel surtout qu'on se retrouve pour chaque sens que l'on pourrait avoir distingué avec des profusions de sens plus ou moins indistinguables les uns des autres.

Un tel déploiement n'a cependant en soi rien d'excessif : il est en définitive assez normal que d'une façon ou d'une autre, quoique d'une manière nécessairement imparfaite, partielle, sur laquelle on va avoir l'occasion de revenir, le champ du sens recouvre le champ indéfini de tout ce que l'on pourrait avoir à dire, à penser, ou simplement à percevoir, champ dont on ne voit pas pourquoi il serait a priori limité, et dont on conçoit qu'il ne soit pas toujours bien distinct. 
Reste que paramétrer ce déploiement indéfini peut sembler impensable, comme si c'était le monde entier, ou l'espace indéfini des pensées, voire l'espace du « tout », qu'il s'agissait de rapporter à quelque faisceau de paramètres.

Il n'est certes pas question de paramétrer tout, bien que ce soit effectivement une propriété du langage que d'être à même si ce n'est de tout dire, du moins de s'employer à tout dire : en l'occurrence, c'est même une propriété particulièrement importante, qui le distingue de beaucoup d'autres pratiques ou activités, et qu'une caractérisation du sens linguistique doit nécessairement prendre en compte.

1 Cependant, ce que nous avons à paramétrer est ce que le langage élabore, et non pas ce qui se trouve être l'objet de cette élaboration. Il est question seulement de paramétrer ce qu'il y a de spécifique à ce sens que le langage déploie à coups d'énoncés : ce qui fait justement que ce sens est élaboration langagière, et n'est pas le tout que par ailleurs, de fait, le langage ne renonce en général pas à essayer de dire. Car, nécessairement, en s'appliquant à le dire, il déploie autre chose : du sens, des sens, qui sont des constructions linguistiques.

\subsection{Des sens caractérisables}

5 Déterminer les paramètres qui organisent le sens au sein des énoncés, c'est soutenir d'abord que ce champ des sens possibles procède de "caractéristiques essentielles » qui peuvent être « explicitées ». Et c'est supposer ce faisant que les sens possibles ne sont pas strictement quelconques, qu'il est possible non pas seulement de les lister, mais aussi de les caractériser. C'est supposer que l'ensemble des sens possibles peut être défini sinon en extension (par une liste), du moins en intension : par ses propriétés caractéristiques ${ }^{12}$. Des propriétés caractéristiques qui figurent ainsi l'invariant se retrouvant dans tous les sens en question: un invariant par delà la variation, mais surtout, on le voit bien ici, un invariant qui est ce qui circonscrit cette variation, puisqu'il est ce par quoi l'ensemble est défini.

53 Cela vaut pour l'ensemble des sens possibles, comme une forme de condition de ce que peut être un sens : on soutient ainsi que les sens possibles répondent nécessairement aux caractéristiques invariantes en question. Mais cela vaut aussi pour tout sens singulier, dont on soutient qu'il n'est justement pas seulement singulier, mais qu'il est rapportable à de telles caractéristiques invariantes. Le sens est caractérisable : le sens en général l'est - ce sont les conditions pour être un sens ; tout sens singulier l'est - le sens d'un énoncé singulier n'est pas une donnée individuelle qu'il suffirait d'enregistrer, il a des propriétés qui les caractérisent, dans sa singularité, et que l'on peut restituer.

Il faut bien voir que ces deux propriétés ne sont pas triviales. On ne les retrouverait pas nécessairement par exemple pour les productions artistiques ; il n'est pas évident que l'on puisse reconstituer les conditions nécessaires et suffisantes pour être une œuvre artistique (ce qui ne veut pas dire que n'importe quoi puisse être une œuvre artistique ; cela veut simplement dire que ce n'est pas sur des conditions que l'être artistique d'une œuvre se détermine); et il n'est pas évident qu'une œuvre artistique soit une entité susceptible d'être rapportée à des caractéristiques. On ne les retrouve certainement pas pour l'ensemble des entités qui constituent ce « tout » que le langage s'échine à dire.

Il n'est certes pas de sémantique qui ne considère que le sens dont elle traite est caractérisable, puisque c'est très précisément son objet que de le caractériser. Mais elle y 
parvient souvent au prix d'une réduction drastique du champ des sens considérés : les sémantiques de manière générale aiment à se constituer dans les frontières de ce qu'elles considèrent pouvoir exclure de leur champ, pragmatique, référence, stylistique, effets subjectifs, figements, connotations, valeurs non vériconditionnelles, sens autres que le sens propre retenu, fioritures intraduisibles - la liste est longue des exclus par les uns et/ ou les autres.

En fait, cette question des frontières est d'abord l'une des raisons pour lesquelles la sémantique forme un programme de recherche fragile, et toujours menacé13 : un programme inauguré seulement à l'aube du 20 ème siècle, alors que la question du sens est bien évidemment présente et centrale dans toutes les réflexions qui se mènent sur le langage, sur la pensée et sur le monde depuis toujours, que ce soit au titre de la philosophie ou à un autre titre; un programme dont l'inauguration n'empêche pas que, jusqu'aux années 70 au moins, on éprouve des difficultés à le mettre en œuvre, avec souvent une tendance à réduire son domaine à la seule sémantique lexicale, parce que le reste précisément ne serait pas caractérisable ${ }^{14}$; un programme qui ne cesse d'être menacé par cette intrusion dans le sens linguistique de ce qui serait son extérieur, avec lequel pourtant il ne cesse de se confondre, et dont il faut sans fin le distinguer, référent, concept, réalité, contenu de pensée, effet d'usage, valeur d'emploi, empan pragmatique, etc.

La sémantique est fragile entre autres parce qu'elle rencontre les plus grandes difficultés pour séparer le sens linguistique qui est son objet, du grand domaine des pensées, des idées, de la connaissance et du monde que ce sens recouvre. C'est là ce qui travaille et ce que fait travailler le texte de Benveniste(1958)sur les «Catégories de langue et catégories de pensée »; on trouve aussi une jolie analyse de ces interférences indéfiniment inextricables dans le Que sais-je de Sémantique de Tamba-Mecz(2007), où elle montre cette incessante échappée du sens hors des langues, dont attestent les paraphrases, traductions, reformulations, et hors du langage aussi bien quand le sens s'identifie avec ce à quoi il renvoie, dans le grand monde des choses et des idées. Car il n'y a pas que le langage et les pensées qui font sens : le monde aussi, les faits, les événements, les rapports entre éléments, le réel des choses, tout cela a du sens, ou en tous les cas peut en avoir, tout cela peut faire sens, sans pour autant que qui que ce soit doive être là pour penser ce sens et encore moins pour être à même de le dire. C'est même après ce sens-là, y compris lorsqu'il est non-sens, que les langues courent, et que les penseurs courent, pour l'exprimer, et pour le penser, y compris lorsqu'il est inexprimable, ou impensable, ce qu'il est nécessairement, au moins en partie : on ne peut pas dire tout ce qui fait sens, donne sens, est le sens; on ne peut pas tout dire, ni tout penser, ce qui implique qu'il faille toujours dire et penser encore - telle est la loi du sens, la loi qui scelle et détermine sans doute l'entreprise cognitive, et qui détermine certainement l'entreprise langagière ${ }^{15}$.

La question de la caractérisation du sens linguistique se pose par conséquent de la façon suivante : le sens linguistique, élaboration langagière, tend en tout point à être indistinct des pensées et du monde que d'une façon ou d'une autre il recouvre. Pourtant, pensées et mondes ne sont pas nécessairement intégralement caractérisables. Et s'ils le sont, il n'y a pas de raison qu'ils le soient par les mêmes biais. Car c'est en tant qu'élaboration langagière que le sens linguistique est caractérisable: la caractérisation en question se fonde sur ce qu'est le langage, ce qu'est un énoncé, ce qu'est une structure linguistique. Or, monde et pensées ne sont pas des faits de langage: le monde n'est pas un fait de langage même si le langage ne cesse de construire des faits de réel (ne serait-ce que les 
paroles sont des faits du monde construits par le langage) ; et bien que le langage bien sûr ne cesse de construire de la pensée, les pensées non plus ne sauraient être réduites à être un fait de langage, comme le fait bien entendre déjà Benveniste(1958)lorsqu'il conclut son analyse en rappelant que les langues tout en déterminant les conditions de la pensée ne sauraient en limiter l'essor. Monde et pensées ne sauraient dès lors s'aligner sur une caractérisation linguistique.

La difficulté est que, s'il faut caractériser le sens linguistique sans en restreindre le domaine, il faudrait pouvoir expliciter ce sens sans l'amputer de cette indistinction remarquable avec pensées et monde, qui lui sont extérieurs, mais qu'il ne cesse de poursuivre et/ou de recouvrir. On reviendra sur la façon particulière dont cette difficulté peut être envisagée par la théorie culiolienne. Le point ici est simplement d'avoir pris la mesure de la difficulté en question, et d'avoir pu ainsi vérifier la non trivialité de l'ambition de caractérisation, du moins lorsqu'on ne s'autorise pas au nom de cette caractérisation à restreindre le champ du domaine qui est à caractériser.

Caractériser le sens linguistique sans pour autant le réduire dans sa dimension propre, et en donnant acte à cette forme d'indistinction avec ce qui lui est extérieur mais qui le détermine dans son déploiement même : tel est le défi qui doit être celui d'une entreprise de paramétrisation du sens linguistique. Parce qu'il s'agit bien évidemment d'embrasser ce sens linguistique dans toute sa dimension. Et parce qu'il s'agit de tenir compte de cette relation à l'extérieur qui le détermine.

\subsection{Syntagmes et paradigmes comme moteurs de diversification du sens}

61 Les premiers moteurs de diversification du sens linguistique, qui induisent sinon toutes les valeurs possibles, du moins toutes celles qui constituent le système d'une langue donnée, sont ceux des relations syntagmatiques et des relations paradigmatiques (ou « relations associatives » pour reprendre les termes du Cours de Linguistique Générale) que Saussure d'abord, les structuralistes ensuite, ont identifiées comme étant les deux types de relations structurant une langue.

Sans doute ces moteurs-là concernent-ils le sens des unités d'une langue, sens qu'il n'est pas évident de concevoir comme un sens étant « élaboré », alors que le titre de cet article annonce une analyse du sens élaboré, qui plus est « au sein des énoncés ».

63 Pourtant, les sens indéfiniment variés qui ont été évoqués ci-dessus et que l'on a vu recouvrir monde et pensées, paraissent ressortir quant à eux à tous les niveaux de l'étagement des productions linguistiques, et non pas au seul niveau des énoncés quel que soit ce niveau. On verra ci-dessous à caractériser en propre ce que peut élaborer un énoncé effectif, qui se distingue de fait de ce qui peut s'élaborer à d'autres niveaux, et en particulier au niveau des unités de la langue, morphèmes ou mots.

Reste qu'à partir du moment où l'on admet que syntagmes et paradigmes déterminent la valeur des unités minimales d'une langue, c'est bien que d'une certaine façon au moins on considère que cette valeur aussi est "élaborée ». Et bien que le CLG ait fait entendre comment la notion de syntagme peut s'appliquer à l'intérieur de la langue (il y a des relations syntagmatiques qui structurent la langue, indépendamment des syntagmes que peuvent ou non construire les énoncés effectifs qu'un locuteur peut produire), il demeure 
qu'à travers au moins ces relations syntagmatiques, il y a une part de la valeur des unités qui peut mobiliser les relations s'établissant au sein d'un énoncé donné.

On va soutenir ici non seulement que tout sens linguistique doit s'appréhender comme étant un sens élaboré, mais encore que d'une façon à préciser l'élaboration s'opère toujours au sein d'énoncés. Sans doute est-ce là ce qui fait de l'approche ici présentée une approche que l'on peut dire «énonciative » : l'énonciation est partout, constitutive de la valeur de chaque unité de langue.

Pour l'entendre, il faut d'abord admettre que les valeurs puissent être « construites » ou "élaborées » sans que ces participes doivent prendre nécessairement une interprétation de type passif, avec quelqu'un qui construit et quelqu'un qui élabore. Y compris pour l'énoncé, parler de construction n'implique pas nécessairement que l'on veuille mobiliser un sujet agent qui ait opéré cette construction : non pas qu'il puisse y avoir d'énoncé sans quelque instance qui l'ait agencé et en ce sens construit, mais parce que le fait qu'une instance - être de chair, institution ou machine - l'ait construit, n'implique pas nécessairement que cet énoncé soit de quelque manière subjectif et doive de toute nécessité être rapporté à une subjectivité ${ }^{16}$.

67 Les énoncés sont construits au sens où ils mobilisent d'autres entités linguistiques, de niveaux différents ou de même niveau, à partir desquels ils tirent leur valeur : l'énoncé Paul dort mobilise des mots et des marques et des lexèmes (Paul, dor-, - $t$ ), et aussi les énoncés environnants sans doute, et aussi le type de discours dans lequel il s'inscrit. Et de la même façon, tout discours, toute proposition, tout syntagme, tout mot constitué de plusieurs composants, est construit. Mais aussi, n'importe quel composant, aussi minimal soit-il, prend une valeur qui est construite parce qu'elle dépend des éléments environnants d'une part (comme l'a mis en évidence l'hypothèse distributionnaliste) et parce qu'elle dépend au moins aussi crucialement de l'unité de niveau supérieur dans laquelle le composant s'intègre (comme l'a mis en évidence Benveniste 1962 dans son fameux article sur les "Niveaux de l'analyse linguistique » où il développe ce concept d' «intégration», pour le donner comme étant ce qui fonde l'identité des unités linguistiques).

68 Cette valeur dépend des éléments environnants et de l'unité intégrante effectivement présents dans telle ou telle production, mais elle dépend aussi des éléments virtuels et de l'unité virtuelle qu'elle appelle, de par les relations syntagmatiques qui se tissent au sein de la langue même.

69 A tous les niveaux par conséquent, le sens est ainsi « élaboré », c'est-à-dire ici construit, à cause au moins des relations syntagmatiques de tous niveaux qui tissent et structurent les langues, avant que de constituer les discours effectifs qu'un locuteur peut produire.

Or c'est là un moteur de diversification des sens qui est clairement illimité, puisque par delà les relations syntagmatiques qui peuvent définir l'unité en langue, les contextualisations effectives de telle ou telle unité sont de fait en nombre illimité. Elles sont en nombre illimité, mais néanmoins contraintes d'une façon ou d'une autre par le faisceau de relations qui caractérise l'unité en langue: dès lors, toutes ces valeurs que chaque unité va prendre d'un contexte à l'autre doivent de quelque façon se rapprocher les uns des autres pour se réduire aux quelques grandes valeurs qu'un dictionnaire par exemple finit par répertorier pour chaque unité. On retrouve bien l'idée annoncée au départ de profusions de sens pourtant plus ou moins indistinguables les uns des autres.

On va voir que la confusion est du même ordre du coté des relations paradigmatiques. 
72 Il faut entendre d'abord que les relations paradigmatiques ressortissent elles aussi d'une élaboration du sens. soutenu le Saussure du CLG: ainsi la valeur d'une flexion casuelle dépend-elle de l'ensemble des ses concurrents potentiels; ainsi la valeur d'un mot comme refaire ou enseignement dépend-elle de la "constellation infinie » des termes qui se trouvent avoir quelque chose en commun, à un titre ou un autre, avec lui. énoncés ou discours, qui prennent leur valeur aussi du réseau des possibles auxquels ils s'opposent et auxquels ils font écho: et le fait est que les deux exemples refaire et enseignement évoqués dans le CLG sont deux mots construits, dérivés, ce qui fait bien entendre que le rôle des relations associatives dans la détermination des valeurs ne concerne pas les seules unités minimales.

Construit par la langue, ou plus précisément par les paradigmes dans lesquels les unités de tout niveau se trouvent prises : on conçoit que le sens ainsi produit soit de ce fait un sens élaboré, en entendant par là au moins qu'il est complexe et qu'il fait intervenir des éléments variés.

76 On peut considérer cependant aussi qu'il est élaboré dans un sens plus dynamique au sein de ces paradigmes : car le fait est que ces derniers peuvent varier et qu'ils varient, au fil du temps, mais aussi d'un parler à l'autre, d'un registre à l'autre, d'un genre à l'autre, et même d'un rapprochement singulier à l'autre, rapprochement singulier effectué par un penseur singulier qui dans un discours singulier aura jugé bon d'inscrire telle nouvelle unité dans tel nouveau paradigme. On sait bien que ces variations, loin d'être marginales, sont constantes, et constitutives de la structure langagière même.

77 Ainsi, tel mot, comme le mot enseignement, va voir sa valeur nourrie par tous les parlers qui l'utilisent et le relient à des pratiques, à des conceptualisations, à des réalités qui peuvent être complexes et variées.

78 Telle marque flexionnelle aussi va voir sa valeur modifiée au fil des bougés dans les paradigmes flexionnels de la langue, que ce soit à l'occasion de variations diachroniques ou des variantes en synchronie qui s'observent dans le domaine flexionnel bien plus largement sans doute que ce que les présentations grammaticales veulent bien l'annoncer. On le sait assez bien pour ce qui concerne le domaine des personnes, puisqu'il est admis que les personnes vont varier selon les types de discours, ou aussi selon les registres (nous de majesté, vous de politesse, on familier, ça populaire, il «impoli », il hypocoristique, etc.). On le sait encore mieux pour les temps-aspects-modes qui varient non seulement selon les parlers, selon les genres, selon les types de discours, mais aussi selon les coups de force de chacun, que l'on retient plus lorsqu'ils se sont écrits, pour de grands innovateurs au travers desquels la langue ne cesse de se construire : on pense ici par exemple aux fameux imparfaits de Flaubert, parmi tous les faits de style que la stylistique a pu noter, mais sans doute aussi à tant et tant d'autres emplois, si tant est qu'il est vrai de ce point de vue que le style est affaire d'écarts (mais d'écarts qui font la langue : la langue aussi est faite d'écarts). Il est évident que cela doit valoir aussi pour toutes les autres marques, cas compris (penser ici au datif éthique, qui est d'un certain registre, et qui fait bouger le paradigme des flexions casuelles du français), genre et nombre compris (ce que l'on entend par exemple au travers des contrastes entre ciels et cieux, ou entre directeur de recherche et directrice d'école). 
79 Et cela vaut clairement pour les unités construites, quand telle locution, mais aussi tel énoncé, voire tel discours, voient leur valeur varier au fil du temps, ou d'un registre à l'autre, parce qu'ils ne sont pas pris dans les mêmes paradigmes.

On retrouve à nouveau le foisonnement annoncé en 2.1. : pas de limites définies, et des valeurs qui peuvent se trouver indéfiniment différenciées, d'une mise en relation paradigmatique à l'autre.

81 Que ce soit pour les relations paradigmatiques ou pour les relations syntagmatiques, on peut douter que l'élaboration des valeurs telle qu'elle vient d'être décrite puisse être ramenée à un calcul que l'on pourrait expliciter : les éléments en jeu sont trop nombreux et indéfiniment variables pour qu'il soit possible par quelque calcul de les intégrer tous à chaque instant, unités de même niveau présents dans le contexte, unités de niveau supérieur déterminant l'ensemble, réseaux de concurrence se déployant diversement au gré des variations tant diachroniques que synchroniques.

Or même en bloquant les effets de diversification et en ne considérant que ce que le CLG appelait un "état de langue » (ce qui peut s'entendre comme une coupure sur l'axe diachronique mais aussi par rapport à toutes les variations d'un discours à l'autre, d'un parler à l'autre et d'un genre à l'autre), Saussure dit lui-même que c'est à des constellations « infinies » que l'on a à faire ; et cet état de langue est un système, ce qui veut dire que la valeur de chaque unité dépend de l'ensemble de ses concurrentes. Si les constellations infinies sont en outre à chaque instant variables, avec des branches propres à un genre, ou à un parler, comme il ressort de la prise en compte des genres, des parlers et des innovations, s'il n'y a que des systèmes mêlés, ce sont des facteurs non seulement indéfiniment variés dans leur contenu mais aussi dans leurs poids respectifs qu'il faut intégrer. La valeur de chaque terme est comme l'ouragan déclenché par la fameuse aile de papillon : on pourrait calculer, en intégrant tous ces facteurs et toutes leurs pondérations, la façon dont cette aile-là plutôt qu'une autre a déclenché cet ouragan-là plutôt qu'un autre, mais pour chaque aile, le calcul serait différent et indéfiniment complexe. Il vaut mieux en rendre compte autrement, procéder de manière plus inductive: déterminer les paramètres à partir desquels ce fouillis se déploie -la forme du déploiement (des syntagmes, des paradigmes) d'une part, ce qui doit être fixé en amont d'autre part pour que ce déploiement soit sinon calculé du moins caractérisé.

Affiner notre compréhension des modalités de déploiement des syntagmes et des paradigmes est un objectif de la caractérisation: les réseaux paradigmatiques sont de diverses natures, comme l'indique déjà la différence faite par le CLG lui-même entre réseaux grammaticaux, fondés sur des relations différentielles, et réseaux lexicaux, en constellations; leurs déploiements passent par des procédés variables que l'on peut tenter de caractériser; et sur les structures syntagmatiques qui peuvent participer à déterminer la valeur des unités, les avancées ont été considérables dans différents champs théoriques, au travers distributions, sous-catégorisations, collocations, ou constructions. De ce point de vue, l'école culiolienne a surtout développé des hypothèses sur les modalités de développement du répertoire syntagmatique d'une unité, avec en particulier une distinction entre trois types de relations entre unités qui sont l'ajout, le nouage et la greffe (voir Paillard 2000). Le développement des paradigmes est cependant l'un des chantiers importants actuels, avec un travail sur les niveaux de langue, les mélanges de langues, la constitution de terminologies d'une part (voir Camus 2009), sur les changements catégoriels d'autre part (voir les différents articles de Camus \& de Vogüé 2004). 
L'autre objectif est d'arriver à déterminer les paramètres en amont qui permettent de caractériser le déploiement en question pour chaque unité examinée : sens propre, sens premier, matrice de sens sont insuffisants s'ils ne parviennent qu'à caractériser l'unité et non pas le déploiement de sa variation; ce que l'on doit déterminer est bien ce que Culioli appelle invariant, intégrant la variation, intégrant toutes les constructions syntagmatiques possibles pour cette unité-là, toutes les mises en réseaux possibles d'une telle unité. On va voir en 2.4. comment à la suite de Benveniste, on a pu proposer de procéder pour reconstituer ces invariants.

\subsection{Les formes schématiques comme paramètres}

85 On se souvient que Benveniste, dans les "Niveaux de l'analyse linguistique », définit le sens des unités linguistiques comme étant leur "capacité intégrative", capacité à intégrer le niveau supérieur, mais justement capacité à intégrer ce faisant toutes les variations que ce niveau va induire: ce qui fait l'identité d'une unité signifiante est sa capacité intégrative. Cette capacité, si l'on parvient à en déterminer la forme précise, doit être le paramètre en amont cherché à partir duquel on caractérise la variation.

Il est frappant que lui-même la décrive comme une « fonction propositionnelle » : car cela veut dire ${ }^{17}$ qu'il la décrit en utilisant la référence même de ce qui a servi à Frege d'exemple type pour définir l'enjeu de l'idéographie, à savoir la fonction, celle pour l'écriture de laquelle il importe plus de marquer la parenthèse que le $x$ variable, celle dans laquelle le $x$ ne vaut que par la position qui est la sienne, que marque la parenthèse. La capacité intégrative dont parle Benveniste est formelle au sens de Frege: c'est une écriture. Elle relève ainsi très exactement de l'entreprise de paramétrage telle que nous l'avons caractérisée en 1.

87 J'ai soutenu que la théorie culiolienne des formes schématiques peut s'interpréter comme une mise en œuvre de ce programme ${ }^{18}$. Chaque unité s'y trouve identifiée par une écriture donnant la forme schématique des différents paramètres que l'unité mobilise. C'est bien de paramètres qu'il est question: des entités à fixer pour que la valeur se déploie; des entités dont l'écriture précise et contraint la forme et les relations qui les lient. Ainsi pour l'unité fil-(présente à la fois dans le nom fil, dans le verbe filer et sans doute dans toutes les unités construites à partir de ceux-ci), ai-je proposé l'écriture suivante : $\mathrm{x}$ est la manifestation continuée en un point $\mathrm{P}$, d'un parcours $\mathrm{X}$ qui constitue le mode d'être Y d'y.

Dans cette formulation dont chaque terme est discutable, c'est sur la donnée des paramètres notés par des lettres que l'analyse s'engage : la prédiction est qu'à chaque fois que fil-se trouve mobilisé dans un texte ou une parole, ces paramètres-là seront mis en place, présents dans le contexte, ou sinon installés du fait même de l'apparition du mot un filant $\mathrm{y}$, avec son mode d'être, un parcours $\mathrm{X}$ qui le constitue, $\mathrm{x}$ qui est le fil, et qui est la manifestation de ce parcours $\mathrm{X}$, et enfin un point $\mathrm{P}$ où cette manifestation aurait $\mathrm{pu}$ s'interrompre et où elle continue.

89 C'est dans cette mesure que l'analyse donne à voir: elle rend perceptible les différents paramètres que l'unité fil- mobilise.

90 Et par ailleurs, elle formule aussi précisément que possible les relations entre ces paramètres, relations qui sont en l'occurrence cruciales puisque ce sont elles qui caractérisent chaque paramètre, mais qu'il faudrait pouvoir seulement écrire au lieu de 
devoir utiliser pour les nommer des mots d'une métalangue toujours insuffisamment dégrossie: sur ce plan, l'objectif est donc bien d'arriver à cette maigreur que Frege exigeait ; la forme que doit prendre cet amaigrissement est encore une question, nous y reviendrons.

\subsection{De l'unité au dire : l'énoncé au moteur du sens}

La forme schématique a une autre particularité importante qui est sa structure prédicative : ce qui se manifeste dans le fait que c'est sous la forme d'un énoncé qu'elle se trouve formulée. De fait, derrière tout emploi de l'unité fil-, il y a l'énoncé d'une manifestation dont il est prédiqué qu'elle est continuée.

Déjà, lorsque Benveniste parle de "fonction propositionnelle» pour caractériser l'identité des unités, quand n'importe quel niveau supérieur intégrant l'unité, n'importe quel syntagme, auraient pu être invoqués, ce sont les propositions que l'unité permet de construire qui sont là seules retenues.

On peut minimiser cette intrusion de la proposition, considérer que c'est seulement la référence à la logique qui l'a induite, parce qu'en logique, les fonctions sont propositionnelles; le fait est que le seul exemple que donne Benveniste(1962) d'unité significative dans l'article est celui d'un nom, salle, et pour illustrer la capacité intégrative de ce nom, on n'a pas de propositions, mais des collocations comme salle d'eau.

On peut la minimiser ou la prendre au sérieux, en retirant à la notion de proposition son sens syntaxique pour retenir ce qu'elle met en jeu: ce qui est en jeu n'est pas tant la nature syntaxique exacte de ce dans quoi l'unité s'intègre, que le fait que ce soit ce que l'on peut dire avec cette unité, ce qui peut s'énoncer avec elle. Il s'agit donc de s'en tenir à un statut plus logique de la proposition: ce qui peut être proposé, ce qui peut être prédiqué $^{19}$, ce qui peut être dit, par exemple pour l'unité salle qu'elle est d'eau. On peut considérer que cela implique au fond d'avoir caractérisé l'identité de l'unité par ce qui de cette unité peut s'énoncer ${ }^{20}$ : ce qui revient à mettre l'énoncé au cœur du sens.

Ainsi retrouve-t-on l'hypothèse évoquée au début de 2.3. et qui soutient la référence aux énoncés dans le titre de cet article: hypothèse selon laquelle c'est toujours «au sein d'énoncés » que le sens s'élabore, y compris lorsque c'est du sens d'une unité qu'il s'agit et pas seulement du sens de l'énoncé lui-même.

Identifier une unité par la forme d'énoncé que cette unité permet de déployer, tel serait donc le programme benvenistien pour déterminer les paramètres de l'élaboration du sens. Et ce serait ces énoncés virtuels que les formes schématiques schématisent: les énoncés qui sont derrière les mots.

\subsection{Des énoncés pour dire : le paramètre du référent}

Reste que le sens s'élabore aussi au sein d'énoncés effectifs, et qu'il faut aussi prendre la mesure de cette élaboration-là. Prendre la mesure de ce qui distingue un énoncé de tout autre syntagme. Ne serait-ce que pour comprendre aussi ce que sont les énoncés virtuels que déploient les formes schématiques.

On a caractérisé les énoncés virtuels à l'œuvre dans les formes schématiques en parlant de prédication et en évoquant le dire que cette prédication mobilise. Cela fait dans tout énoncé actuel autant d'énoncés virtuels qu'il y a d'unités lexicales. La question alors est 
de comprendre ce qui dans ce déploiement virtuel permet de caractériser malgré tout un énoncé actuel donné.

Empiriquement, on reconnaît l'énoncé actuel au fait que ce soit une séquence linguistique qui d'une façon ou d'une autre se donne comme étant complète, suffisamment complète en tous les cas pour que le locuteur qui le profère puisse considérer qu'il peut si besoin laisser la parole : un énoncé est la forme minimale qui peut constituer un tour de parole.

Cela reste compatible avec des structures syntaxiques variables: un mot simple peut servir d'énoncé, une suite de mots inachevée, une phrase elliptique, peuvent servir d'énoncé. Ces structures sont non quelconques et il revient aux syntacticiens de les étudier, mais elles sont variables. Reste que sur le plan sémantique, il y a énoncé dès lors que cette structure s'entend comme formant de quelque façon un tout.

Or, ce qui donne la mesure de ce tout, est qu'il y ait eu quelque chose à dire. Non pas que ce quelque chose soit dit, au sens du moins où il serait tout dit, intégralement dit, ce qui ne saurait être possible. Mais que la séquence linguistique se soit employée à dire quelque chose qu'elle avait à dire.

On propose cette mesure-là au dire, une mesure rétroactive : il y avait quelque chose à dire. C'est ce qui permet à la séquence linguistique de se constituer comme étant un dire. On voit que cela correspond d'une certaine façon au thème de l'énoncé. Tant du moins que l'énoncé peut être divisé entre thème et propos. Or on sait qu'il peut y avoir des structures d'énoncé différentes, lorsque par exemple l'énoncé est un énoncé narratif comme "Un homme entra ", dans lequel il n'y pas de sens à distinguer un thème, parce que tout l'énoncé a pour propos de construire le référent dont il parle. On sait aussi qu'il y a des énoncés plus complexes, avec des thèmes qui sont déjà des propos, propos secondaires, au moins d'un certain point de vue : par exemple dans des énoncés avec des descriptions définies qui ne sont pas seulement déterminatives mais déjà prédicatives ("L'idiot comprit »). Présent ou absent de l'énoncé, distinct ou identique aux différents propos de l'énoncé, ce que l'énoncé s'est donné à dire correspond en fait, plutôt qu'au thème, à ce que l'énoncé se donne comme référent : un référent qu'il va nommer ou non et il y a thème s'il le nomme -, un référent qu'il s'emploie de toutes façons à décrire, au travers des prédications opérées.

Ce référent qui suffit à constituer comme un tout l'énoncé qui le dit, est un référent visé par le dire, ce qui fait toute la différence avec les référents d'unités autres : l'énoncé est pris dans une dynamique, induite par le fait que son référent soit ainsi à dire. Pour faire entendre cette dynamique, on parle donc de "référent à dire », ou simplement de "à dire $»^{21}:$ ce qui fait l'énoncé effectif, c'est cet « à dire » qui le détermine.

\subsection{Entre dit et à dire : l'épaisseur du sens}

Si l'analyse qui vient d'être proposée de ce qui se joue au sein d'un énoncé est correcte, cela signifie que la mesure de ce qu'est un énoncé se trouve précisément à l'extérieur du linguistique : du coté de ce qu'il y a à dire, des pensées, du monde, du référent.

On a indiqué en 2.1. le problème posé par la confusion caractéristique de l'activité linguistique, entre ce que le langage élabore d'une part et ce sur quoi porte cette élaboration d'autre part, qui pourtant lui est extérieur.

Cette difficulté tient sans doute, de manière générale, au fait que les élaborations langagières se donnent comme des représentations de ce sur quoi elles portent; en tant 
que représentations, il n'est pas anormal qu'elles puissent prétendre prendre la place du représenté, celui-ci se trouvant ainsi littéralement re-présenté à travers elles, présent sous la forme que peuvent lui donner ces représentations.

Cependant, dans le cas des énoncés, occupés à dire, et constitués comme dires à partir de cet «à dire » qui les fonde, il n'y a pas simplement représentation : car ce que l'énoncé construit comme représentation et ce qu'il est occupé à représenter, qui est cet «à dire ", sont à la fois nécessairement séparés, et constitutivement inséparables ${ }^{22}$.

Et la raison de cette confusion est que, pour l'énoncé, représentant construit et représenté figurent l'un et l'autre ce que l'énoncé dit : ce que l'énoncé élabore est ce qu'il dit ; l'objet de cette élaboration est ce dont il parle, mais peut aussi être décrit comme étant ce qu'il dit. Et de fait ils peuvent tout deux être désignés comme tels.

Il y a là une propriété essentielle du verbe dire, dont l'objet peut renvoyer selon les cas soit à ce dont on parle, et que l'on cherche à dire, soit à ce que l'on dit effectivement, et donc à ce qui résulte du dire : voir dire son bonheur, son exaspération, qui peut signifier chercher des mots pour exprimer ce bonheur ou cette exaspération que l'on a à dire, mais qui peut signifier aussi formuler, via des mots, que l'on est heureux et exaspéréz

110 Cette propriété particulière du verbe dire, loin d'être un simple effet de brouillage, est à la mesure de ce qui soutient l'activité même du dire, prise entre deux visées, à la fois distinctes (faute de quoi le dire n'est rien) et pourtant tenues pour équivalentes (faute de quoi le dire a échoué) : d'un coté le «à dire ", qui n'est justement pas dit tant du moins qu'on n'a pas réussi à le dire, et qu'il y a tant de façons de dire; de l'autre le dire ou plus exactement ce que ce dire veut dire, qui est ce qu'il dit effectivement, et qui n'est qu'une représentation de ce qui était à dire.

Que le dire et le à dire jamais véritablement ne se séparent donne à ce sens à la fois un caractère clivé et une épaisseur : son sens n'est jamais simplement ce qu'il dit, mais ce qu'il dit en relation avec ce qu'il s'est donné à dire. On va voir en 2.8. que cette épaisseur est d'autant plus importante qu'elle peut se trouver matérialisée par des couches en nombre indéfini venant remplir la distance du à dire au dit.

112 Mesurer la distance du dire au à dire, prendre la mesure de cette mise en coïncidence qui se construit entre dit et à dire au fil du dire, entre une valeur référentielle construite, celle du dire, et le référent qui est à dire : tel est l'enjeu d'une caractérisation du sens qui rende compte effectivement de la façon dont il s'élabore. Il faut alors pouvoir faire le départ entre dire et à dire. Cela dépend de la façon dont le dire se structure, entre dire du à dire et dire de ce qu'il y a à dire de ce à dire, entre thème et propos, désignation et prédication, selon qu'ils se distinguent ou se confondent.

113 Cette structure, c'est l'analyse syntaxique des structures prédicatives de l'énoncé qui peut en donner la mesure. Encore faut-il qu'au lieu de réduire tout énoncé à une seule structure, bipartite avec un thème et un propos, ou plate avec toutes les unités référant à égalité à une situation de référence qu'elles recouvrent, l'analyse syntaxique veuille bien entendre la pluralité des structurations possibles selon que chaque unité est elle-même prise comme une prédication ou comme une désignation par exemple. Cette pluralité des syntaxes, ainsi que ce jeu entre prédication et désignation, est l'un des objets sur lesquels la Théorie des Opérations Prédicatives et Enonciatives fait des propositions en distinguant en particulier entre une référence quantitative de type désignation et une référence qualitative de type prédication, et en déployant surtout toute une grammaire 
des différentes pondérations possibles entre référence quantitative et référence qualitative ${ }^{24}$.

Mais au point de départ de ces différents calculs syntaxiques, caractérisant le dire qu'ils peuvent élaborer, il y a d'une part tous les paramètres que chaque unité mobilisée déploie au travers de sa forme schématique, et il y a d'autre part le référent à dire autour duquel le dire en question s'organise.

On voit par conséquent en quoi consiste le paramétrage du sens dans le cas de l'énoncé : développer ces différents paramètres, et développer la façon dont ils s'organisent entre eux -la façon dont ils se combinent, la façon dont ils se trouvent pris dans la structure de l'énoncé, rapportés pour les uns à une simple désignation du référent, pour les autres ou les mêmes à une prédication sur ce référent.

Il s'agira d'une caractérisation, il ne s'agira pas d'un calcul. Parce que la valeur de chaque unité ne saurait être calculée ainsi qu'on l'a vu en 2.3., parce que la façon dont ces unités se combinent ne ressortit pas non plus à un calcul mais plutôt à une description ainsi qu'on l'a vu à la fin de 2.3., et parce que, comme on va le voir en 2.8 et 2.9., le sens obtenu est pris à son tour dans un foisonnement de sens possibles, en fait un double foisonnement, de sens qui sont à la fois en nombre illimité, et tous en partie indistinguables de celui-là.

\subsection{Troisième moteur de déploiement du sens : les gloses du dire}

117 Caractériser à coups de paramètres et de modalités de structuration la façon dont l'épaisseur du sens se déploie entre dire et à dire, est une chose. L'expliciter par des gloses aussi précises et rigoureuses que faire se peut en est une autre, qui est possible mais qui est proprement sans fin, et qui prendra nécessairement des formes en nombre indéfini. Car en explicitant, on ne pourra pas faire l'économie de devoir dire ce que le dire veut dire, engendrant alors une démultiplication des niveaux, propre à matérialiser encore plus l'épaisseur du sens, ainsi qu'on l'annonçait, mais qui tournera au vertigineux quand il faudra distinguer entre ce que veut dire le dire et ce que ce « vouloir dire » à son tour peut vouloir dire.

118 Cette démultiplication passe entre autres par l'explicitation de ce que chacun des éléments dits veut dire: elle a alors pour ressort les réseaux paradigmatiques et les intégrations syntagmatiques dont il a été question en 2.3., et c'est tout le foisonnement de la langue qui peut ainsi venir nourrir ce que l'énoncé a dit.

119 Elle passe aussi par l'explicitation des relations entre dire et à dire et des différentes instances qui peuvent être mobilisées pour articuler l'un à l'autre: entre ce que le locuteur qui a dit voulait dire, ce que veut dire ce qu'il a dit, ce que veulent dire les mots et phrases qu'il a produits, ce que veut dire aussi ce qui était à dire, mais aussi entre ce que tout cela dit, et encore entre ce que tout cela signifie, ce qui n'est pas forcément pareil, et ce que tout cela implique, ce qui est encore autre chose.

C'est ce déploiement que de manière très empirique rencontre tout locuteur, dès lors qu'il se trouve à devoir expliciter des énoncés, que ce soit les siens propres ou ceux qui se présentent à lui et qu'il prétend interpréter : «Qu'est-ce que tu/je veux dire par ce que tu/je dis? ", ce qui suppose que ce que tu/je dis, que je comprends puisque je comprends cette langue, puisse ne pas suffire à dire ce que tu/je veux dire ; mais encore "Qu'est-ce que tu/je cherche(s) à dire ? », qui suppose que ce que tu/je dis soit distinct de ce que tu/ 
je cherche(s) à dire ; et alors aussi «Qu'est-ce que très exactement tu/je en dis ?»; et même "Que veut dire ce qu'ainsi tu/je en dis ?", quand c'est la langue elle-même qui du coup demande à être explicitée ; et ensuite « Que veut dire au fond ce que tu/je cherche(s) à dire? ", quand il a fallu passer par le sens de ce qui est à dire pour trouver celui de ce qui en est dit; et parfois «Que pense(s)-tu/je/tel ou tel, de ce que cela veut dire et/ou de ce que cela dit et/ou de ce qui est à dire? ", jusqu'à y compris « Qu'est-ce que tu/je/tel ou tel, pense(s) de ce qu'il faut en dire ? ", «Qu'est-ce qu'il faut en dire ? », voire « Qu'est-ce qu'il faut dire? ?, ou encore "Qu'est-ce qu'on peut dire?».

Chacun de ces feuillets du dire, d'une interprétation à l'autre, d'un paradigme à l'autre, d'un effet de contexte à l'autre, d'un point de vue à l'autre, d'une velléité à l'autre, d'une légitimité à l'autre, travaille à éclairer les autres. Mais chacun, du même mouvement, vient marquer la part d'opacité des autres, égale à ce qui reste de leur différence, qui n'a pas été explicité par le feuillet en question. Ainsi, c'est aussi le caractère indicible de ce qui est à dire (que le dire n'a pu dire), mais aussi de ce que veut dire le dit (qu'il faut pourtant redire), et au final de chacun des feuillets considérés, qui se trouve au cœur du travail d'explicitation, ou qui en est le ressort, qui fait qu'il faille toujours redire ce que rien ne peut avoir dit exactement.

Cela explique qu'il puisse y avoir dans cette quête du sens à la fois des affaires éventuellement graves de partage de légitimité, et des questions insondables relatives au mystère de ce qui ne peut se dire et qui irrémédiablement reste indéchiffrable. Cela explique les forts enjeux qui peuvent être attachés à ce travail de glose et de reformulation, quand il porte sur quelque différend entre personnes, ou, encore plus fortement, quand il porte sur la façon d'interpréter tel énoncé de vérité, loi, dogme, mythe, règle de vie, ou écriture sainte.

C'est pour décrire ce travail de reformulation que Culioli a proposé de parler d'activité épilinguistique, activité que tout locuteur ne se prive pas de pratiquer, qui est elle-même productrice de dires en nombre indéfini, et qui se trouve par conséquent démultiplier d'autant le champ des dires dont on se donne pour tâche de caractériser le sens.

On voit que c'est là un troisième moteur, propre aux énoncés effectifs celui-là, de déploiement du sens, puisqu'à chacune des gloses produites pour expliciter le sens, s'attache un nouveau sens, à la fois distinct du premier et indistinguable de celui-ci. Le sens linguistique en est d'autant plus foisonnant, qui non seulement prétend recouvrir le « tout » du monde comme on l'a rappelé en 2.1., mais intègre aussi l'ensemble indéfini des reformulations que l'on peut produire pour redire chaque dire. Il est par conséquent deux fois infini : recouvrant les sens indéfiniment variés du monde infini, et recouvrant en chaque point toutes les gloses en nombre indéfini qui pour chaque sens peuvent être produites pour le dire.

On conçoit que dans ce double foisonnement, il vaille mieux caractériser le sens de tel dire singulier plutôt que d'envisager de le calculer. Quitte à le caractériser en s'appuyant sur ce que les paquets de gloses permettent d'expliciter : voir de Vogué (2001) pour une explication de la façon dont les gloses peuvent aider à rendre visible ce qui dans un énoncé s'opère, et une application au cas des énoncés conditionnels.

\subsection{Quatrième moteur : les paraphrases}

Il y a un quatrième moteur au déploiement indéfini des sens, avec de nouveaux paquets de dires possibles, engendrant de nouvelles formes d'indistinguabilité. Des paquets que 
l'on pourrait confondre avec les paquets de gloses qui viennent d'être pris en considération, dans la mesure où il s'agit toujours de différentes façons de dire la même chose, mais qui méritent néanmoins d'en être différenciés parce qu'ils ne procèdent pas des mêmes mécanismes : il s'agit des séries de paraphrases qui peuvent être produites pour formuler un même dire. La paraphrase existe. Elle est même abondante. Il n'y a pas d'énoncé qui ne puisse donner lieu à des paraphrases, et ces paraphrases sont pour chaque énoncé en grand nombre, sinon en nombre illimité. Ainsi, pour ne citer qu'un cas bien connu, une interrogation comme "Où es-tu? " peut être paraphrasée par toutes les formes possibles d'interrogation, formes dont on ne sait pas forcément trop ce qui les différencie, mais qui sont nombreuses et peut-être indéfiniment diversifiables en droit, dans la mesure où certains des procédés qu'elles mettent en jeu peuvent, en droit, être indéfiniment réitérés : "Où tu es?", «Tu es où ?", «Où est-ce que tu es?", « Où c'est que tu es? ", « Où est-ce que c'est que tu es? ", « Où c'est que c'est que tu es? ", "C'est où que tu es? " "C'est où que c'est que tu es?", sans compter les «Où t'es ti ? » et autres "Où c'est ti que tu es? ", et encore "Où peux-tu donc bien être? ", "Où peux-tu bien être ?", «Où peux-tu être ? ", « Où est-ce que tu peux être ? ", etc.

La différence avec les gloses est qu'il ne s'agit pas d'expliciter: il s'agit simplement d'autres chemins prenant d'autres détours (ou plus de détours) pour dire le à dire. Les paraphrases ne sont pas plus explicites les unes que les autres, ou du moins ce n'est pas sur ce principe qu'elles se diversifient; elles utilisent seulement d'autres moyens. Alors que les gloses, longues, abstraites, constituent une forme d'énoncés tout à fait particulière, propre au gloseur, et assez différente du langage ordinaire, les paraphrases sont des phrases ordinaires, qui se trouvent simplement avoir des valeurs suffisamment proches pour qu'on puisse les tenir pour à peu près équivalentes, au moins d'un certain point de vue.

Sans doute n'y a-t-il jamais de paraphrase exacte, et la différence de sens, même si on ne sait pas l'expliciter, est bel et bien présente, au moins parce que les formes ne sont pas les mêmes, ce qui signifie que le tour suivi n'est pas le même. Il demeure que la relation de paraphrase existe, au sens où deux phrases en relation de paraphrase ne sont pas dans la même relation que deux phrases qui ne diraient pas la même chose d'un certain point de vue.

L'existence d'une telle relation dans les langues est au demeurant l'une des propriétés remarquables du langage, par rapport à d'autres systèmes de signification, qui ne présentent généralement pas un tel luxe de moyens : pourquoi tant de façons de dire la même chose, quand une seule pourrait suffire si c'était seulement ce que l'on dit qui importait? Précisément, l'existence des paraphrases montre que dans le langage il n'y a pas que ce que l'on dit qui importe, mais aussi la façon dont on le dit: l'existence des paraphrases montre que la "façon" entre à part égale avec le contenu dans la détermination du sens linguistique.

Une des particularités des paraphrases qui les distingue des gloses, outre leur caractère ordinaire, est qu'elles puissent donner lieu à des limitations : ainsi à coté de "Je pense bien!" pour marquer que quelque chose est vrai, on a "Je crois bien!", mais à coté de «Tu penses bien!», on n'aura pas « Tu crois bien!». Ou plutôt, on l'aura mais avec une autre valeur qui n'est plus en relation de paraphrase avec la phrase de départ. Dans toutes les séries paraphrastiques on trouve de tels impossibles ${ }^{25}$. Cela ne se produit pas avec les gloses parce que les gloses ne relèvent pas d'opérations particulières que l'on ne pourrait pas mettre en œuvre dans certaines conditions. 

paraphrases relèvent à chaque fois d'opérations d'agencement particulières: des opérations qui peuvent être caractérisées comme toute opération syntaxique, par ce qui constitue leur conditionnement. C'est la raison pour laquelle on a pu faire des paraphrases la base empirique de l'étude des agencements syntaxiques : on se souvient le rôle qu'elles ont joué aussi bien dans les grammaires chomskiennes que dans les grammaires harrissiennes, quand Chomsky définissait les opérations syntaxiques (comme le passif, l'interrogation ou l'extraction, entre autres opérations centrales de la syntaxe) par les couples de paraphrases que chacune pouvait produire, ou quand Harris définissait à rebours une structure syntaxique par une classe de paraphrases possibles pour cette structure. parce que précisément pour lui la syntaxe loin d'être une instance de régulation des formes observables, est une instance de déploiement des formes dans leur foisonnement. Parce que son objet n'est pas de voir ce qui régule ces formes, mais de déterminer les paramètres qui rendent compte de ce foisonnement. Dès lors, pour déployer ses familles paraphrastiques, il ne se prive pas d'ajouter des éléments (il ajoute donc, bien, c'est que, peut). Non pas pour voir comment cela se compose, mais plutôt pour voir ce que cela produit comme déformation: comment le résultat se trouve perturbé, comment néanmoins il se restabilise. extérieur $\mathrm{E}$, avec au point de bifurcation la possibilité de considérer la valeur et son extérieur, pour maintenir les deux, pour n'en retenir qu'un en excluant l'autre, pour ignorer l'autre, pour envisager l'autre comme une alternative excluant la valeur de départ, etc. (voir Culioli 1988)

137 Ainsi, pour reprendre l'exemple des formes d'interrogation, ce qui serait en jeu dans ce déploiement-là, et que marqueraient diversement les opérations que sont l'inversion, l'extraction, la pronominalisation, la modalisation par pouvoir, etc., ce serait les différentes façons de prendre en compte les alternatives à la réponse cherchée. Les nombreux autres cas de quasi paraphrases qui sont étudiés au sein de l'école culiolienne 
mettent tous en jeu d'une façon ou d'une autre ce travail sur l'altérité (voir par exemple Culioli 1981).

Là encore, il ne saurait y avoir calcul pour rendre compte de chaque paraphrase. Il s'agit simplement de trouver les bons paramètres pour déployer le champ des paraphrases possibles, et pour rendre ce faisant perceptible le travail qui est effectué sur l'altérité.

\section{Conclusion}

On a des paramètres pour caractériser, parce que l'on exclut de pouvoir calculer étant donné le foisonnement des formes et des valeurs à caractériser. On utilise justement ces paramètres pour expliquer comment les formes et les valeurs se diversifient: certainement pas pour réduire ce foisonnement, et le ramener à quelque régularité plus calculable, mais au contraire pour le déployer et en rendre compte. On utilise ces paramètres pour donner à voir ce déploiement du sens : donner à voir ce dont il est fait, rendre perceptible la façon dont il se constitue et ce qui l'induit.

Ces paramètres, on en a mentionné quelques-uns au long de notre parcours des différents moteurs $\mathrm{du}$ foisonnement: les paramètres des formes schématiques, le paramètre $d u$ référent à dire, le paramètre de l'altérité. Ils sont sommaires. Ils peuvent paraître trop sommaires pour rendre compte de tels foisonnements. Le fait est que pour en rendre compte, ils s'associent à tout ce qui a pu être évoqué par ailleurs concernant les modalités de différenciation des paradigmes, les modalités de construction de syntagmes, les modalités de structuration de l'énoncé, les modalités de prise en compte de l'autre. Reste que leur caractère sommaire même est ce qui permet aussi à la diversité de se déployer aussi largement. L'ambition pour ces paramètres est justement que ce soit ce que l'on a appelé dans la première partie des lettres maigres: suffisamment maigres et sans substance propre pour pouvoir intégrer tous les déploiements.

On a vu à propos de la forme schématique de fil- que sa formulation restait provisoire, parce que les termes utilisés semblaient quant à eux posséder trop de substance propre pour être assurés de dire exactement les relations entre les paramètres mobilisés. Il ne s'agit pas de trouver un métalangage rigoureux qui parvienne à recouvrir exactement les valeurs: un tel métalangage n'existe pas, comme en atteste la profusion des reformulations épilinguistiques. Il s'agit de trouver les termes à fixer à partir desquelles les valeurs de fil- dans chaque emploi pourront s'induire. Des termes comme parcours sont trop lourds encore de leur substance propre, sauf à avoir réussi à les rendre opératoires. C'est sur cela que le travail de formulation se concentre: voir deVogüé(2001) pour la façon dont il a pu être mené dans le champ de l'expression de la condition.

De la maigreur donc, qui ne permettra certes pas d'expliquer pourquoi on a tel sens singulier pour tel énoncé singulier. On explique pourquoi il y a ce foisonnement de sens pour l'ensemble des énoncés. On n'explique pas chaque sens singulier. Parce que ces sens singuliers ne demandent pas à être expliqués. Ils demandent à être constatés, reconnus. Ils demandent à être rendus perceptibles. Et en particulier à être discernés d'autres sens singuliers d'énoncés proches voire a priori indistinguables.

143 C'est dire que le programme que l'on se donne est d'abord descriptif : arriver à discerner ce qui se joue dans un énoncé, arriver à discerner des sens qui d'un certain point de vue paraissent indistinguables. Le sens est à lire, il n'est pas tout lu. Il n'est pas une donnée 
brute. Pour arriver à le lire, il faut avoir pris les bonnes lunettes, le bon observatoire, les bonnes mesures : il faut avoir pu se donner en amont les bons paramètres.

\section{BIBLIOGRAPHIE}

Auchlin, A., Moeschler, J. (1997) Introduction à la linguistique contemporaine, Cursus Armand Colin. Benveniste, E. (1958) « Catégories de langue et catégories de pensée », Problèmes de linguistique générale, 1, TEL Gallimard, 1966, p. 63-74.

Benveniste, E., (1959) «Le système des temps dans le verbe français », Problèmes de linguistique générale, 1, TEL Gallimard, 1966, p. 237-250.

Benveniste, E. (1962) «Les niveaux de l'analyse linguistique », Problèmes de linguistique générale, 1, TEL Gallimard, 1966, p. 119-131.

Camus, R.Site INVLEX, http://camus.remi.free.fr/INVLEX/.

Camus, R. (2009) « Du slavon en russe contemporain : caractérisation générale et l'exemple de da à valeur optative ». In : J. Breuillard (éd.) Mélanges en l'honneur du professeur Jean-Paul Sémon, Paris, Institut d'Etudes slaves.

Camus, R., de Vogüé, S., (2004) «Variation sémantique et syntaxique des unités lexicales : étude de six verbes français ", LINX $50: 7-13$.

Carbonnel, J. P. (1995) « Paramètre et variable », C.N.F.S.H., http://91.121.162.160/glu/FRDIC/DICPARAM.HTM.

Cori, M. (2003) « La mathématisation des formalismes syntaxiques », LINX 48 : 13-28.

Culioli, A. (1981) « Sur le concept de notion », in Pour une linguistique de l'énonciation 1, Ophrys, 1990, pp. 47-65.

Culioli, A. (1987) « La linguistique : de l'empirique au formel », in Pour une linguistique de l'énonciation 1, Ophrys, 1990, pp. 9-46.

Culioli, A. (1982) « Le rôle des représentations métalinguistiques en syntaxe », in Pour une linguistique de l'énonciation 2, Ophrys, 1999, pp. 95-106.

Culioli, A. (1988) « Autres commentaires sur bien », in Pour une linguistique de l'énonciation 1, Ophrys, 1990, pp. 157- 168.

De Vogué, S. (2001) «L'épilangue au pied de la lettre. A propos du concept grammatical de condition ", Le gré des langues 16, L'harmattan, Paris, pp. 9-48.

De Vogué, S. (2004) «Syntaxe, référence et identité du verbe filer », LINX 50 : 135-167.

De Vogué, S. (2006a) «Invariance culiolienne : l'exemple du passé, entre passés, parfaits et passages », in Ducard D., Normand C. (eds), Antoine Culioli, Un homme dans le langage, Colloque de Cerisy, Ophrys, pp. 302-331.

De Vogué, S. (2006b) « Les principes organisateurs de la variété des constructions verbales », non publié, mis en ligne sur le site LLF. 
De Vogué, S. (2011), « L'énonciation dans le lexique. Actualité du concept benvenistien d'intégration dans la théorie des formes schématiques de l'école culiolienne ». In : Brunet E., Mahrer R. (eds) Relire Benveniste. Réceptions actuelles des Problèmes de linguistique générale. Louvainla-Neuve, Collection « Sciences du langage : carrefours et points de vue », Academia- Bruylant, pp. 169-195.

Franckel, J.-J. (1998) « Au cœur de l'indicible : le verbe dire », in Franckel, J.-J., et Normand, C. (eds) L'indicible et ses marques dans l'énonciation, Numéro spécial de LINX, Université Paris X Nanterre, pp. 53-70.

Frege, G. (1882) "Que la science justifie le recours à une idéographie », in Ecrits logiques et philosophiques, trad. C. Imbert, Paris, Seuil, 1971, p. 63-69.

Frege, G. (1904) « Qu'est-ce qu'une fonction », in Ecrits logiques et philosophiques, trad. C. Imbert, Paris, Seuil, 1971, pp. 160-169.

Lacan, J. (1975) Encore, Le séminaire, Livre XX, Seuil.

Lakatos, I. (1970) « Falsification and the methodology of scientific research programmes », in I. Lakatos et A. Musgrave (eds), Criticism and the Growth of Knowledge, Londres, Cambridge University Press.

Milner, J.C. (1989) Introduction à une science du langage, Paris, Seuil.

Paillard, D. (1997) «Dire Staline », Le gré des langues 11, L'Harmattan, pp. 68-91.

Paillard, D. (2000), « A propos des verbes « polysémiques » : identité sémantique et principes de variation », Syntaxe et sémantique, 2, pp. 99-120.

Russell, B. (1919) Introduction à la philosophie mathématique, trad. G. Moreau, Paris, Payot, 1970.

Saussure, F. de (1915) Cours de Linguistique générale, publié par Bally C., Sechehaye, A., Payot, Paris, 1969.

Tamba-Mecz, I. (2007) La sémantique, Collection Que sais-je ?, PUF.

\section{NOTES}

1. Cette seconde perspective n'est pas différente dans l'esprit de celle qu'a déployée en particulier la grammaire générative autour du programme dit des Principes et Paramètres, quand il s'agissait de déterminer les paramètres qui ordonnent les différences entre langues et permettent d'en rendre compte à partir de ce qui serait une grammaire universelle : simplement le programme serait appliqué non pas à la grammaire des langues mais aux configurations sémantiques qu'elles déploient, dont on admettrait qu'elles aussi pourraient être paramétrées.

2. Voir par exemple Culioli (1987), ou de Vogüé (2006a).

3. Cette efficacité se mesure au fait que l'on puisse changer de paramètres, ou choisir de meilleurs paramètres, qui seront meilleurs parce qu'ils explicitent d'une manière plus parlante, ou plus manipulable, donc plus efficace, l'ensemble considéré.

4. Voir le principe de composition, rapporté à Frege, selon lequel le tout s'obtiendrait à partir des parties qui le composent. Un principe dont il ne faut pas oublier qu'il est le contrepied de ce qui fonde selon Saussure les relations syntagmatiques (soit l'un des deux axes sur lesquelles les langues s'ordonnent) et la notion même de syntagme, qui est que le tout (le syntagme) ne soit pas la somme des parties. Les deux affirmations sont compatibles si l'on admet qu'il y a d'autres façons de composer des parties que celle consistant à en faire la somme, façons que la sémantique formelle s'est de fait employée à identifier. Il demeure que l'assertion de Saussure, 
qui ne donnait peut-être pas au terme de somme un sens restreint, fragilise le principe de composition.

5. Ce qui la caractérise « littéralement » justement. Car c'est bien d'un ordre littéral qu'il s'agit, et il faut là entendre la notion de lettre dans ce sens de littéralité, qui est le sien dans l'expression «à la lettre » par exemple : littéraliser l'empirie serait inscrire, à la lettre, littéralement, ce que cet empirie est littéralement; ce serait restituer ce qui fait sa littéralité. Sur la lettre quelques éléments supplémentaires dans de Vogüé(2001).

6. On précise là « sujet agent », plutôt que simplement « sujet », parce qu'il n'est pas impossible qu'au travers de cette écriture sans agent s'écrive un sujet, pure instance subjective, prise dans les réseaux du subjectif et de l'intersubjectif, mais non pour autant agent, agissant, maître de ce qui s'est écrit.

7. Voir les malheurs du mauvais mathématicien, qui face aux écritures mathématiques, répète qu'il ne " comprend » pas, quand c'est seulement lorsqu'il consentira à renoncer de comprendre qu'il pourra alors entendre - ce qui s'écrit : renonce à essayer de comprendre, écris, est-on tenté de lui dire, sachant bien que pour résoudre un problème mathématique complexe, il faut d'abord se contenter d'écrire le problème, pour que la voie de solution alors, comme à notre insu, se dessine. Ce travail de soustraction du sujet-agent par rapport à ce qu'il écrit est sans aucun doute au fondement de la pratique des lettres en général, du mathématique comme comble de la lettre en particulier.

8. Un chemin déductif pourrait être parcouru rétroactivement, de l'empirique vers ses paramètres constituants, cet empirique étant le résultat d'un calcul à partir des paramètres en question, et donc se déduisant intégralement. Le chemin inductif ne peut lui être remonté.

9. En fait Lakatos quant à lui conserve la réfutabilité, faisant seulement entendre que dans la mesure où il n'existe pas de fait brut, non théorisé, on ne réfute pas une théorie par un fait mais par une autre théorie, celle qui théorise le fait avancé en réfutation de la théorie de départ : d'où l'idée selon laquelle c'est fondamentalement par la critique des théories existantes que la connaissance progresse. Cependant, pour que l'empirique reste le moteur de cette progression, il faut que le fond de la critique ne porte pas sur la théorisation donnée des mêmes faits, mais sur le type de faits que chaque théorie permet de voir : la meilleure théorie est celle qui voit le plus de faits, qui voit des faits invisibles pour d'autres théories. Dès lors la mesure de la bonne théorie n'est plus sa réfutabilité mais sa capacité à percevoir des faits. Et les théories se conçoivent comme des programmes de description de l'empirique, proposant des outils d'observation. A ce moment-là, la question de la vérité se trouve remplacée par une problématique de la perceptibilité, et les constituants de la théorie sont moins des propositions que des outils, ou si l'on veut sont des propositions d'existence qui disent ce qu'il y a et non pas des propositions attributives qui qualifient ce qui est. On voit que cela rejoint très exactement ce qui est dit ici sur le paramétrage.

10. Sur le plan méthodologique, les conséquences sont importantes: cela signifie pour le linguiste qu'il ne s'appuie plus, ou du moins plus crucialement, sur les contrastes (oppositions ou paires minimales), et sur les conditions d'acceptabilité. Il faut donc changer d'observatoire : la glose, que l'épistémologie culiolienne a replacée au centre de l'activité du linguiste, est cet observatoire nouveau qui vise à rendre explicite ce qui est dans le sens, et non pas seulement à le contraster, et non pas seulement pour l'opposer à ce qui n'est pas. On va y revenir plus loin dans le texte. Ne plus se centrer sur les paires minimales et la recherche d'inacceptables, se concentrer sur la recherche des contextes qui permettent de déployer les gloses, et sur la recherche des paraphrases qui attestent de la non-différenciabilité qui ordonne le langage : c'est cette autre pratique de la linguistique que je revendique depuis des années contre le mot d'ordre du différentialisme.

11. Ainsi des trois caractéristiques que Cori(2003) considère comme étant exigées de toute mathématisation, sa falsifiabilité, sa prédictivité, son objectivité, on ne retient que la troisième, 
que Cori explicite d'ailleurs en faisant lui aussi référence à une affaire de mains : «mises entre n'importe quelles mains, les mêmes hypothèses dansun même modèle doivent conduire aux mêmes conclusions » (p. 15). La falsifiabilité est remplacée par un discernement qui n'a rien de différentiel, qui est strictement perceptif : il s'agit de rendre visible et perceptible l'empirie. La prédictivité du calcul déductif est remplacée par le déploiement inductif d'un invariant, qui ne prédit rien, mais qui caractérise.

12. Pour mémoire, on pense ici à la différence entre une définition en extension et en intension de l'ensemble des nombres pairs par exemple: en extension, on doit en donner la liste, infinie $(\{0 ; 2 ; 4 ; 6$; etc. $\})$; en intension on donne la propriété caractéristique qui conditionne l'appartenance à l'ensemble (ce qui peut s'écrire $\{x / x$ est un nombre pair $\}$ ).

13. Sans doute y a-t-il d'autres raisons, commela peur que peut susciter la question du sens. On pense à la description lyrique qu'en fait Benveniste(1962) où le sens est comparéà une hydre. Sans doute y a-t-il aussi le vertige, dont on donnera une idée plus loin dans le texte : un vertige que produit la perspective d'avoir à aller jusqu'à donner le sens du sens.

14. Il se trouve que ce rejet de la question sémantique s'est trouvé explicitement revendiqué lors de mon premier contact avec la linguistique alors que j'étais étudiante: l'enseignante au demeurant remarquable dont je suivais le cours d'initiation a cru bon d'affirmer que la linguistique serait devenue une science à partir du moment où elle a bien voulu se débarrasser de la question du sens; elle soutenait là une position sans doute bien peu saussurienne, mais totalement en phase avec le distributionnalisme ou avec la thèse de l'autonomie de la syntaxe qui s'étaient déployés aux alentours du milieu du siècle.

15. Bien évidemment, cette loi du " pas-tout » n'est pas à prendre comme une théorie mystique, mais bien comme une position épistémologique, quels que soient les effets de mysticisme qu'elle engendre. Sur le pas-tout, voir en particulier Lacan (1975).

16. Si la catégorie de l'Histoire existe, telle que l'a caractérisée Benveniste (1959), elle correspond clairement à un type d'énonciation qui ne se rapporte pas à une subjectivité.

17. Malgré le fait que Benveniste fasse là explicitement référence à Russell : par delà les oppositions sur lesquelles on s'est appuyé dans la première partie, il demeure que Russell comme Frege travaillent à l'élaboration de la fonction propositionnelle, l'un plus occupé par les variables, l'autre plus occupé par les parenthèses.

18. Voir de Vogüé(à paraître).

19. Voir la caractérisation que Benveniste donne plus loin dans l'article de qu'il appelle alors phrase, donnée alors comme premier niveau d'un tout autre univers, l'univers du discours, dont ce qui est ici appelé énoncé serait clairement la clef.

20. Noter que le même problème semble être à l'origine de ce qui s'est appelé le lambda-calcul, au moins tel que l'a utilisé Montague, lorsqu'il a s'agi d'intégrer toutes les prédications qui s'opèrent dans une proposition syntaxique, non seulement celles marquées par le prédicat verbal, mais aussi celles qui se trouvent impliquées par les noms et les adjectifs, chacune étant alors notée par une fonction lambda, qui permet de rapporter l'unité aux prédications qu'elle permet d'opérer. Que les prédications soient partout dans les phrases, déterminant chacun des items lexicaux en présence, et non pas seulement le prédicat verbal, telle est l'évidence que rencontre l'élargissement de la logique des prédicats au lambda-calcul, l'opérateur lambda étant ce qui permet de révéler la nature prédicative de tout item. Pour un aperçu du lambda-calcul, voir Auchin \& Moeschler(1997).

21. Parler de « à dire ", c'est se placer dans la suite de l'un des gestes fondateurs de Culioli quand il substitue au dictum latin donnant le contenu du dire, son "dit», la lexis grecque définie justement comme «dicible» ou «à dire ", et entendue non plus comme point de stabilité mais comme génératrice de dires. Noter que la lexis culiolienne se caractérise aussi par le fait qu'elle s'ordonne sur un schéma, dit justement schéma de lexis, schéma qui organise l'énoncé (le même schéma que ceux des formes schématiques organisant l'énoncé virtuel que l'unité déploie), quand 
le dictum est matériau brut que le dire façonne. Lalexis façonne le dire. Voir Culioli(1981) ou Culioli(1982).

22. D'où les difficultés signalées ci-dessous à distinguer clairement dans chaque énoncé entre un thème d'une part, un propos d'autre part. D'où les difficultés aussi à distinguer dans le cas des énoncés entre référent et sens, difficulté dont l'une des manifestations aigues a pu être la décision frégéenne de rapporter le référent des propositions à tout autre chose que le contenu de pensée qu'elle pouvait véhiculer et en l'occurrence à une valeur de vérité.

23. Voir pour une analyse de toutes les possibilités Paillard(1997) et Franckel(1998).

24. Voir Culioli(1981) et deVogué(1986b) en particulier.

25. Noter que ce ne sont pas des impossibles en soi, mais simplement des impossibles pour une paraphrase : ce qui a été dit ci-dessus contre les abus de la méthodologie des inacceptables n'est pas ici fondamentalement remis en cause.

\section{RÉSUMÉS}

On remet en cause les principes de prédictivité et de falsifiabilité appliqués à l'analyse sémantique et on propose une démarche inductive fondée sur la détermination des paramètres à partir desquels le sens linguistique se déploie. On soutient que le sens linguistique ressortit, à tous les niveaux de l'analyse linguistique, d'une élaboration énonciative. On montre que ces élaborations procèdent d'un déploiement illimité, induit par les quatre moteurs de diversification que sont les syntagmes, les paradigmes, les gloses et les paraphrases. On propose une caractérisation de la forme énoncé articulant référent à dire et valeur référentielle construite. On évoque un certain nombre de paramètres à l'œuvre dans le déploiement de sens, au travers des formes schématiques qui caractérisent l'identité de toute unité lexicale, au travers des jeux de mise en coïncidence entre référent extérieur et valeur référentielle, au travers des jeux sur l'altérité qui travaillent à un titre ou un autre toute construction de l'identité.

This paper discusses the principles of predictivity and falsifiability applied to semantic analysis and proposes an inductive approach based on parameters involved in the unfolding of meaning. It claims that linguistic meaning emerges out of 'enunciative' elaborations at every level of linguistic analysis. These elaborations proceed from unlimited development, induced by four sources of diversification: syntagms, paradigms, reformulations and paraphrases. A characterization of the utterance (seen as the French "énoncé") is proposed here, based on the articulation between the reference to be and the referential value it elaborates. Some parameters involved in the construction of meaning are discussed: what is described as "schematic forms" characterizing the identity of any lexical unity, but also the different modalities of articulation between referent and referential value, as well as inter-subjective positioning processes, which are bound to be involved in any construction of identity.

\section{INDEX}

Mots-clés : paramètre, forme schématique, épistémologie, élaboration du sens

Keywords : parameter, schematic form, epistemology, meaning construction 


\section{AUTEUR}

SARAH DE VOGÜÉ

MoDyCo, UMR 7114, Université de Paris Ouest La Défense 